UDK 528.73

\title{
DISCUSSION ON THE ORTHOMETRIC HEIGHT REALIZATION
}

\author{
Robert Tenzer \\ School of Civil Engineering and Geosciences, University of Newcastle upon Tyne, \\ Cassie Building, Newcastle upon Tyne, United Kingdom, NE1 7RU, \\ e-mail: robert.tenzer@ncl.ac.uk;
}

Received 1207 2004; accepted 03012005

\begin{abstract}
In the theory of the orthometric height, the mean value of gravity along the plumbline between the geoid and the earth's surface is defined as the integral mean. To determine the mean gravity from the gravity observations realized at the physical surface of the earth, the actual topographical density distribution and vertical change of gravity with depth have to be known. In Helmert's (1890) definition of the orthometric height, the assumption of the linear change of normal gravity is used adopting the constant topographical density distribution. The mean value of gravity is then approximately evaluated so that the observed gravity of a point at the earth's surface is reduced to the mid-point of the plumbline by Poincaré-Prey's gravity gradient. To avoid the problems related to the determination of mean gravity, Molodensky (1945) formulated the different concept. In his theory of the normal height, the mean value of the normal gravity along the ellipsoidal normal between the ellipsoid surface and telluroid is considered. The mean normal gravity is then evaluated explicitly without any hypothesis about the topographical density distribution and vertical gradient of actual gravity. In this paper, the corrections to Helmert's orthometric height are formulated based on the comparison of the integral mean of gravity and Poincaré-Prey's gravity reduction. As follows from the results of the numerical investigation, the orthometric heights can also be determined with a reasonable accuracy if the sufficient information about topographical density and gravity are available.
\end{abstract}

Keywords: orthometric height, gravity, Poincaré-Prey's gravity reduction.

\section{Introduction}

For a practical realization of the geodetic vertical datum, Helmert's [1] orthometric heights are preferably used. The reason is a simple computation of the mean gravity using Poincaré-Prey's gravity reduction and the acceptable accuracy for most of the regions where the leveling networks are established. To determine the orthometric heights in the mountainous regions with the accuracy of a few centimeters or even better, Helmert's definition is not sufficient. In this case, a more precise method for the evaluation of mean gravity is required.

A more accurate method was introduced by Niethammer [2]. It takes terrain into account, assuming the constant topographical density distribution. The mean value of the planar terrain correction is evaluated as a simple average of values computed at the finite number of points along the plumbline within the topography. Mader [3] estimated the difference between Helmert and Niethammer's methods of about $6 \mathrm{~cm}$ for Hochtor $(2504 \mathrm{~m})$ in the Alps, see also Heiskanen and Moritz (chap. 4-6, [4] ). Mader also presupposed that the terrain correction varies linearly with depth. Based on this assumption, the mean terrain correction is averaged from two values computed for points at the earth's surface and geoid.

It is well known fact that the mean gravity depends on the actual topographical density distribution. The variation of topographical density can cause centimetre and decimetre changes of the orthometric height [5]. The correction to Helmert's orthometric height due to the lateral variation of topographical density can be evaluated using a simple formula in which the change of orthometric height is in a linear relation to the anomalous lateral topographical density at the computation point [4]. Adopting this relation, the effect of the anomalous topographical density to Helmert's orthometric heights was investigated by Allister and Featherstone [6] and Tenzer and Vaníček [7].

In Helmert's definition of the orthometric height, the vertical gradient of gravity generated by the geoid is approximated by the linear change of normal gravity, disregarding the vertical gradient of the geoid-generated gravity disturbance as well as the change of the normal gravity gradient with depth. Hwang and Hsiao [8] estimated that this approximation causes centimetre inaccuracy of orthometric height in the mountains.

The analytical downward continuation of the observed gravity at the earth's surface was used in Tenzer et al. [9] to evaluate the mean gravity. To make the gravity along the plumbline analytical, the laterally varying model of topographical density distribution was adopted. The relation between Poincaré-Prey's gravity gradient and the analytical downward continuation of gravity was then formulated and corrections to Helmert's orthometric height arising from the relation were discussed. More recently, a new method for a determination of the mean gravity along the plumbline 
was introduced in Tenzer et al. [10]. The mean gravity is decomposed into the mean normal gravity, mean gravity disturbance generated by the geoid and mean values of the gravitational attractions of topographical and atmospheric masses. The mean normal gravity is evaluated according to Somigliana-Pizzetti's theory of the normal gravity field [11], [12]. The mean topographygenerated gravitational attraction is, in accordance with Bruns' [13] theorem, defined in terms of the difference of gravitational potentials reckoned to the geoid and earth's surface, multiplied by the reciprocal value of the orthometric height. The same principle is deduced for a definition of the mean atmosphere-generated gravitational attraction. The mean geoid-generated gravity disturbance is defined applying Poisson's integral to the integral mean.

Comparing the integral mean of gravity and Poincaré-Prey's gravity reduction, the corrections to Helmert's orthometric height are formulated and results of numerical investigation discussed in this contribution.

\section{Orthometric height, mean gravity}

The actual length of the plumbline between the geoid of which the geocentric radius is denoted by $r_{g}(\Omega)$ and the earth's surface, $r_{t}(\Omega) \cong r_{g}(\Omega)+H^{\mathrm{O}}(\Omega)$, defines the orthometric height $H^{\mathrm{O}}(\Omega)$, e.g., Heiskanen and Moritz [4], Eq. 4-21

$$
H^{\mathrm{O}}(\Omega)=\frac{C\left[r_{t}(\Omega)\right]}{\bar{g}(\Omega)},
$$

where $C\left[r_{t}(\Omega)\right]$ is the geopotential number, and $\bar{g}(\Omega)$ the mean value of actual gravity along the plumbline within the topography.

To define a position, the system of the geocentric coordinates $\phi, \lambda$ and $r$ is used, where $\phi$ and $\lambda$ are the geocentric spherical latitude and longitude $\Omega=(\phi, \lambda)$, $(-\pi / 2 \leq \phi \leq \pi / 2 ; 0 \leq \lambda<2 \pi)$, and $r$ the geocentric radius $r \in(0,+\infty)$.

The mean gravity $\bar{g}(\Omega)$ along the plumbline in Eq. (1) is given by Heiskanen and Moritz [4], Eq. 4-20

$$
\begin{aligned}
& \bar{g}(\Omega)= \\
& =\frac{1}{H^{\mathrm{O}}(\Omega)} \int_{r=r_{g}(\Omega)}^{r_{g}(\Omega)+H^{\mathrm{O}}(\Omega)} g(r, \Omega) \cos \left(-\mathrm{g}(r, \Omega), \mathrm{r}^{\mathrm{o}}\right) \mathrm{d} r,
\end{aligned}
$$

where $\cos \left(-\mathbf{g}(r, \Omega), \mathbf{r}^{\mathbf{o}}\right)$ is the cosine of the deflection of the plumbline from the geocentric radial direction, and $\mathbf{r}^{\mathbf{0}}$ the unit vector in the geocentric radial direction. As numerically estimated in Tenzer et al. [10], neglecting the deflection of the plumbline from the geocentric radial direction could result in a few millimetres inaccuracy of the orthometric height.
Helmert [1] approximated the mean gravity $\tilde{g}(\Omega)$ using Poincaré-Prey's gravity reduction. It reads [4], Eq. 4-25

$$
\begin{aligned}
& \tilde{g}(\Omega) \cong g\left[r_{t}(\Omega)\right]-\left.\frac{\partial g(r, \Omega)}{\partial \mathrm{t}}\right|_{r=r_{t}(\Omega)} \frac{H^{\mathrm{O}}(\Omega)}{2} \cong \\
& \approx g\left[r_{t}(\Omega)\right]-\left(\left.\frac{\partial \gamma(r, \phi)}{\partial \mathrm{n}}\right|_{r=r_{t}(\Omega)}+4 \pi \mathrm{G} \rho_{\mathrm{o}}\right) \frac{H^{\mathrm{O}}(\Omega)}{2},
\end{aligned}
$$

where $\mathrm{G}$ is Newton's gravitational constant, $\rho_{\mathrm{o}}$ the mean value of topographical density, and $\partial g(r, \Omega) / \partial \mathrm{t}$ and $\partial \gamma(r, \phi) / \partial \mathrm{n}$ represent the actual and normal linear changes of gravity, respectively.

According to Eq. (3), the approximate value $\tilde{g}(\Omega)$ of mean gravity is evaluated so that the observed gravity $g\left[r_{t}(\Omega)\right]$ of a point at the earth's surface is reduced to the mid-point of the plumbline $H^{\mathrm{O}}(\Omega) / 2$. The expression in the brackets on the right-hand side of Eq. (3) represents Poincaré-Prey's gravity gradient, in which the linear change of normal gravity is used, assuming the constant topographical density distribution $\rho_{0}$.

\section{Relation between integral mean of gravity and Poincaré-Prey's gravity reduction}

To find the relation between the integral mean of gravity and Poincaré-Prey's gravity reduction, the actual gravity $g(r, \Omega)$ is decomposed into the normal gravity $\gamma(r, \phi)$, gravity disturbance $\delta g^{N T}(r, \Omega)$ generated by the geoid, and gravitational attractions of topographical and atmospheric masses $g^{t}(r, \Omega)$ and $g^{a}(r, \Omega)$. Thereby [14]

$$
g(r, \Omega)=\gamma(r, \phi)+\delta g^{N T}(r, \Omega)+g^{t}(r, \Omega)+g^{a}(r, \Omega),
$$

where the sum of the normal gravity $\gamma(r, \phi)$ and the geoid-generated gravity disturbance $\delta g^{\mathrm{NT}}(r, \Omega)$ represents the gravity $g^{\mathrm{NT}}(r, \Omega)$ generated by the geoid, $g^{\mathrm{NT}}(r, \Omega)=\gamma(r, \phi)+\delta g^{\mathrm{NT}}(r, \Omega)$.

Since the effect of atmosphere on the orthometric height is negligible [10], the gravitational attraction of atmospheric masses is not considered in the sequel of discussion.

By analogy with Eq. (4), the mean gravity $\bar{g}(\Omega)$ in Eq. (2) can be rewritten as

$$
\bar{g}(\Omega) \cong \bar{\gamma}(\Omega)+\overline{\delta g}^{N T}(\Omega)+\bar{g}^{t}(\Omega)
$$

where $\bar{\gamma}(\Omega)$ is the mean value of the normal gravity along the plumbline within the topography, $\overline{\delta g}^{N T}(\Omega)$ 
mean geoid-generated gravity disturbance, and $\bar{g}^{t}(\Omega)$ mean topography-generated gravitational attraction.

Substituting Eq. (4) to Eq. (3), Poincaré-Prey's gravity reduction becomes

$$
\begin{aligned}
& \tilde{g}(\Omega)=\gamma\left[r_{t}(\Omega)\right]-\left.\frac{\partial \gamma(r, \phi)}{\partial \mathrm{n}}\right|_{r=r_{t}(\Omega)} \frac{H^{\mathrm{O}}(\Omega)}{2}+ \\
& +\delta g^{\mathrm{NT}}\left[r_{t}(\Omega)\right]+g^{t}\left[r_{t}(\Omega)\right]-2 \pi \mathrm{G} \rho_{\mathrm{o}} H^{\mathrm{O}}(\Omega),
\end{aligned}
$$

where the observed gravity $g\left[r_{t}(\Omega)\right]$ is divided into the gravity components $\quad \gamma\left[r_{t}(\Omega)\right], \quad \delta g^{\mathrm{NT}}\left[r_{t}(\Omega)\right] \quad$ and $g^{t}\left[r_{t}(\Omega)\right]$ which are reckoned at the earth's surface.

Comparing Eqns. (5) and (6), the following basic relation is introduced

$$
\begin{aligned}
& \bar{g}(\Omega)-\tilde{g}(\Omega) \cong \bar{\gamma}(\Omega)-\gamma\left[r_{t}(\Omega)\right]+\left.\frac{\partial \gamma(r, \phi)}{\partial \mathrm{n}}\right|_{r=r_{t}(\Omega)} \frac{H^{\mathrm{O}}(\Omega)}{2}+ \\
& +\overline{\delta g}^{\mathrm{NT}}(\Omega)-\delta g^{\mathrm{NT}}\left[r_{t}(\Omega)\right]+ \\
& +\bar{g}^{t}(\Omega)-g^{t}\left[r_{t}(\Omega)\right]+2 \pi \mathrm{G} \rho_{\mathrm{o}} H^{\mathrm{O}}(\Omega)
\end{aligned}
$$

As follows from the above equation, the relation between the integral mean of gravity $\bar{g}(\Omega)$ and its approximate value $\tilde{g}(\Omega)$ in Helmert's definition consists of three individual terms related to the components of mean gravity. Investigating these terms, the corrections to Helmert's orthometric height due to the mean normal gravity, mean geoid-generated gravity disturbance and mean topography-generated gravitational attraction are formulated in the next paragraphs. Furthermore, the correction due to the mean topography-generated gravitational attraction is treated separately for terrain roughness and anomalous topographical density.

\section{Correction related to the mean normal gravity}

Applying the analytical downward continuation of normal gravity to the integral mean, the following expression is obtained [9]

$$
\bar{\gamma}(\Omega)=\gamma\left[r_{t}(\Omega)\right]-\left.\sum_{k=1}^{\infty} \frac{1}{(k+1) !} \frac{\partial^{\mathrm{k}} \gamma(r, \phi)}{\partial \mathrm{n}^{\mathrm{k}}}\right|_{r=r_{t}(\Omega)}\left[H^{\mathrm{O}}(\Omega)\right]^{k}
$$

Comparison of the mean normal gravity in Eq. (8) and its counterpart in Poincaré-Prey's gravity reduction (Eq. 6) yields

$$
\begin{aligned}
& \bar{\gamma}(\Omega)-\gamma\left[r_{t}(\Omega)\right]+\left.\frac{\partial \gamma(r, \phi)}{\partial \mathrm{n}}\right|_{r=r_{t}(\Omega)} \frac{H^{\mathrm{O}}(\Omega)}{2}= \\
& =-\left.\sum_{k=2}^{\infty} \frac{1}{(k+1) !} \frac{\partial^{\mathrm{k}} \gamma(r, \phi)}{\partial \mathrm{n}^{\mathrm{k}}}\right|_{r=r_{t}(\Omega)}\left[H^{\mathrm{O}}(\Omega)\right]^{k} .
\end{aligned}
$$

The variation of normal gravity gradient with depth in Eq. (9) can further be rewritten as [4], Eq. 2-121

$$
\begin{aligned}
& \left.\sum_{k=2}^{\infty} \frac{1}{(k+1) !} \frac{\partial^{\mathrm{k}} \gamma(r, \phi)}{\partial \mathrm{n}^{\mathrm{k}}}\right|_{r=r_{t}(\Omega)}\left[H^{\mathrm{O}}(\Omega)\right]^{k} \approx \\
& \left.\approx \frac{1}{3 !} \frac{\partial^{2} \gamma(r, \phi)}{\partial \mathrm{n}^{2}}\right|_{r=r_{t}(\Omega)}\left[H^{\mathrm{O}}(\Omega)\right]^{2} \cong \frac{\gamma_{o}(\phi)}{\mathrm{a}^{2}}\left[H^{\mathrm{O}}(\Omega)\right]^{2},
\end{aligned}
$$

where a is the major semi-axis of the reference ellipsoid, and $\gamma_{o}(\phi)$ normal gravity at the surface of ellipsoid.

The correction $\varepsilon_{H}^{\gamma}(\Omega)$ to Helmert's orthometric height due to the variation of normal gravity gradient can then be found to be [9]

$$
\varepsilon_{H}^{\gamma}(\Omega) \cong \frac{\left[H^{\mathrm{O}}(\Omega)\right]^{3}}{\mathrm{a}^{2}}
$$

This correction reaches up to $13 \mathrm{~mm}$ for the height $8000 \mathrm{~m}$, while for heights up to $3500 \mathrm{~m}$ less than $1 \mathrm{~mm}$.

\section{Correction due to the mean geoid-generated gravity disturbance}

As stated in the Introduction, the geoid-generated gravity gradient in Poincaré-Prey's gravity reduction is approximated by the linear change of normal gravity, disregarding the geoid-generated gravity gradient. Thereby, the correction $\varepsilon_{H}^{\delta g}(\Omega)$ to Helmert's orthometric height due to the mean geoid-generated gravity disturbance is applied. It reads

$$
\varepsilon_{H}^{\delta g}(\Omega)=-\frac{H^{O}(\Omega)}{\bar{g}(\Omega)}\left(\overline{\delta g}^{N T}(\Omega)-\delta g^{N T}\left[r_{t}(\Omega)\right]\right) .
$$

The mean gravity $\bar{g}(\Omega)$ in the denominator of Eq. (12) can be replaced by the normal gravity $\gamma_{o}(\phi)$ without decreasing the numerical accuracy.

The correction to Helmert's orthometric height due to the mean geoid-generated gravity disturbance was numerically investigated for a part of the Canadian Rocky Mountains between $50^{\circ}$ and $55^{\circ}$ of the geodetic latitude and between $235^{\circ}$ and $339^{\circ}$ of the geodetic longitude. At this territory, it varies between -2 and $9 \mathrm{~cm}$. This result is very similar to that presented in [9], in which the corrections were formulated based on the comparison of the analytical continuation of gravity and Poincaré-Prey's gravity gradient. Since similar results were obtained also for the corrections related to the mean topographygenerated gravitational attraction, they are not again graphically interpreted in this paper.

Applying Poisson's upward continuation to the integral mean, the mean geoid-generated gravity disturbance $\overline{\delta g}^{N T}(\Omega)$ in Eq. (12) is found to be [10] 


$$
\begin{aligned}
& \overline{\delta g}^{N T}(\Omega)=\frac{1}{4 \pi} \frac{R}{H^{O}(\Omega)} \times \\
& \times \iint_{\Omega^{\prime}} \int_{r=R}^{R+H^{o}(\Omega)} r^{-1} K\left(r, \Omega ; R, \Omega^{\prime}\right) d r \delta g^{N T}\left[r_{g}\left(\Omega^{\prime}\right)\right] d \Omega^{\prime},
\end{aligned}
$$

where $K\left(r, \Omega ; R, \Omega^{\prime}\right)$ denotes the Poisson kernel. The mean radius of the earth $\mathrm{R}$ [15] in Eq. (13) is used to approximate the geocentric radius of the geoid surface, i.e., $r_{g}(\Omega) \approx \mathrm{R}$. As follows from the above equation, the mean value $\overline{\delta g}^{N T}(\Omega)$ is evaluated by convolution of the radially integrated Poisson's kernel and the geoidgenerated gravity disturbances $\delta g{ }^{N T}\left[r_{g}(\Omega)\right]$ reckoned to the geoid surface.

The geoid-generated gravity disturbances $\delta g^{N T}\left[r_{g}(\Omega)\right]$ in Eq. (13) are computed from the geoidgenerated gravity anomalies $\Delta g^{N T}\left[r_{g}(\Omega)\right]$. In this case, the geoid-generated gravity anomalies $\Delta g^{N T}\left[r_{t}(\Omega)\right]$ at the earth's surface are continued downward onto the geoid solving the Poisson integral equation. Alternatively, the geoid-generated gravity disturbances $\delta g^{N T}\left[r_{t}(\Omega)\right]$ can be downward continued, after they are computed from the geoid-generated gravity anomalies $\Delta g^{N T}\left[r_{t}(\Omega)\right]$ at the earth's surface. Disregarding the ellipsoidal corrections to the fundamental gravimetric equation, the relation between them is given by

$$
\begin{aligned}
& \delta g^{N T}\left[r_{t}(\Omega)\right] \cong \Delta g^{N T}\left[r_{t}(\Omega)\right]-\left.\frac{\partial \gamma(r, \phi)}{\partial n}\right|_{r=r_{o}(\phi)+H^{N}(\Omega)} \zeta(\Omega)- \\
& -\frac{2}{r_{t}(\Omega)} V^{t}\left[r_{t}(\Omega)\right]-\frac{2}{r_{t}(\Omega)} V^{a}\left[r_{t}(\Omega)\right]
\end{aligned}
$$

where $\varsigma(\Omega)$ denotes the height anomaly [16], and $V^{t}(r, \Omega)$ and $V^{a}(r, \Omega)$ are the gravitational potentials of topographical and atmospheric masses.

Furthermore, the geoid-generated gravity anomaly $\Delta g^{N T}(r, \Omega)$ in Eq. (14) is defined so that the effect of topographical and atmospheric masses is subtracted from the actual gravity anomaly $\Delta g(r, \Omega)$. The fundamental gravimetric equation formulated for the geoid-generated gravity anomaly reads [14]

$$
\begin{aligned}
& \Delta g^{N T}(r, \Omega) \cong \Delta g(r, \Omega)-g^{t}(r, \Omega)-g^{a}(r, \Omega)+ \\
& +\frac{2}{r} V^{t}(r, \Omega)+\frac{2}{r} V^{a}(r, \Omega)+\varepsilon_{\delta g}^{N T}(r, \Omega)-\varepsilon_{n}^{N T}(r, \Omega),
\end{aligned}
$$

where $\varepsilon_{\delta g}^{N T}(r, \Omega)$ and $\varepsilon_{n}^{N T}(r, \Omega)$ stand for the ellipsoidal corrections to the gravity disturbance and for the spherical approximation, respectively. The effect of topographical masses on the gravity anomaly consists of the direct and secondary indirect topographical effects. The direct topographical effect is given by the second term on the right-hand side of Eq. (15), and the fourth term represents the secondary indirect topographical effect. Equivalently, the third term stands for the direct atmospheric effect, and the fifth term is the secondary indirect atmospheric effect.

A different concept for a determination of the mean geoid-generated gravity disturbance is available. Since the mean geoid-generated gravity disturbance can be defined in terms of the geoid-generated disturbing gravity potential difference (of two values reckoned to the geoid and earth's surface), the mean value $\overline{\delta g}^{N T}(\Omega)$ can be evaluated from the geoid-generated gravity anomalies $\Delta g^{N T}\left[r_{g}(\Omega)\right]$ solving Stokes' boundary-value problem.

This is allowed due to the well-known fact that Stokes' integral defines the relation between the disturbing gravity potential and gravity anomalies. Regarding that gravity anomalies are used more often in geodetic applications than gravity disturbances, this method is more appropriate for a numerical realization.

\section{Corrections due to the mean topography- generated gravitational attraction}

Comparing the mean value of the topographygenerated gravitational attraction $\bar{g}^{t}(\Omega)$ in Eq. (5) and the corresponding term of Poincaré-Prey's gravity reduction in Eq. (6), the following expression is written

$$
\bar{g}^{t}(\Omega)-g^{t}\left[r_{t}(\Omega)\right]+2 \pi \mathrm{G} \rho_{\mathrm{o}} H^{\mathrm{O}}(\Omega) .
$$

The integral mean of the topography-generated gravitational attraction $\bar{g}^{t}(\Omega)$ in Eq. (16) is given by

$$
\begin{aligned}
& \bar{g}^{t}(\Omega) \cong \frac{1}{H^{\mathrm{O}}(\Omega)} \int_{r=\mathrm{R}}^{\mathrm{R}+H^{\mathrm{O}}(\Omega)} g^{t}(r, \Omega) \mathrm{d} r= \\
& =\frac{V^{t}\left[r_{g}(\Omega)\right]-V^{t}\left[r_{t}(\Omega)\right]}{H^{\mathrm{O}}(\Omega)} .
\end{aligned}
$$

According to Martinec [17], the gravitational attraction of topographical masses $g^{t}\left[r_{t}(\Omega)\right]$ reckoned at the earth's surface reads

$$
\begin{aligned}
& g^{t}\left[r_{t}(\Omega)\right]=4 \pi \mathrm{G} \rho_{\mathrm{o}} \frac{\mathrm{R}^{2}}{r_{t}^{2}(\Omega)} H^{\mathrm{O}}(\Omega)\left[1+\frac{H^{\mathrm{O}}(\Omega)}{\mathrm{R}}+\frac{1}{3}\left(\frac{H^{\mathrm{O}}(\Omega)}{\mathrm{R}}\right)^{2}\right]- \\
& -\left.\mathrm{G} \rho_{\mathrm{o}} \iint_{\Omega^{\prime}} \int_{r^{\prime}=\mathrm{R}+H^{\mathrm{O}}(\Omega)}^{\mathrm{R}+H^{\mathrm{O}}\left(\Omega^{\prime}\right)} \frac{\partial \ell^{-1}\left(r, \Omega ; r^{\prime}, \Omega^{\prime}\right)}{\partial r}\right|_{r=r_{t}(\Omega)} r^{\prime 2} \mathrm{~d} r^{\prime} \mathrm{d} \Omega^{\prime}- \\
& -\left.\mathrm{G} \iint_{\Omega^{\prime}}^{\mathrm{R}+H^{\prime}\left(\Omega^{\prime}\right)} \delta \rho\left(r^{\prime}, \Omega^{\prime}\right) \frac{\partial \ell^{-1}\left(r, \Omega ; r^{\prime}, \Omega^{\prime}\right)}{\partial r}\right|_{r=r_{t}(\Omega)} r^{\prime 2} \mathrm{~d} r^{\prime} \mathrm{d} \Omega^{\prime},(18)
\end{aligned}
$$


where $\ell\left(r, \Omega ; r^{\prime}, \Omega^{\prime}\right)$ denotes the spatial distance. In Eq. (18), the actual topographical density $\rho(r, \Omega)$ is divided into the mean density $\rho_{\mathrm{o}}$ and anomalous density $\delta \rho(r, \Omega)$, so that $\rho(r, \Omega)=\rho_{\mathrm{o}}+\delta \rho(r, \Omega)$.

The first term on the right-hand side of Eq. (18) is the gravitational attraction $g^{\mathrm{SBS}}\left[r_{t}(\Omega)\right]$ of the spherical Bouguer shell, see [18]. The second term stands for the spherical terrain correction $g^{\mathrm{STC}}\left[r_{t}(\Omega)\right]$, i.e., the gravitational attraction of the spherical terrain roughness term [19]. The last term defines the gravitational attraction $g \delta\left[r_{t}(\Omega)\right]$ of the anomalous topographical density distribution, see [20]. Eq. (18) can then be formally rewritten into the following simple form

$$
g^{t}\left[r_{t}(\Omega)\right]=g^{\mathrm{SBS}}\left[r_{t}(\Omega)\right]+g^{\mathrm{STC}}\left[r_{t}(\Omega)\right]+g^{\delta \rho}\left[r_{t}(\Omega)\right] .
$$

Applying the above decomposition, the mean value of the topography-generated gravitational attraction $\bar{g}^{t}(\Omega)$ is divided into the mean value of gravitational attraction $\bar{g} \operatorname{SBS}(\Omega)$ of the spherical Bouguer shell, mean spherical terrain correction $\bar{g}^{\mathrm{STC}}(\Omega)$ and mean value of gravitational attraction $\bar{g} \delta \rho(\Omega)$ of the anomalous topographical density distribution. Thereby

$$
\bar{g}^{t}(\Omega)=\bar{g}^{\mathrm{SBS}}(\Omega)+\bar{g}^{\mathrm{STC}}(\Omega)+\bar{g}^{\delta \rho}(\Omega) .
$$

With reference to Eq. (17), Eq. (20) becomes [10]

$$
\begin{aligned}
& \bar{g}^{t}(\Omega)=2 \pi \mathrm{G} \rho_{\mathrm{o}} H^{\mathrm{O}}(\Omega)\left[1+\frac{2}{3} \frac{H^{\mathrm{O}}(\Omega)}{\mathrm{R}}\right]+ \\
& +\frac{\mathrm{G} \rho_{\mathrm{o}}}{H^{\mathrm{O}}(\Omega)} \iint_{\Omega^{\prime}} \int_{r^{\prime}=\mathrm{R}+H^{\mathrm{O}}(\Omega)}^{\mathrm{R}+H^{\mathrm{O}}\left(\Omega^{\prime}\right)}\left(\ell^{-1}\left(\mathrm{R}, \Omega ; r^{\prime}, \Omega^{\prime}\right)-\right. \\
& \left.-\ell^{-1}\left[r_{t}(\Omega) ; r^{\prime}, \Omega^{\prime}\right]\right) r^{\prime 2} \mathrm{~d} r^{\prime} \mathrm{d} \Omega^{\prime}+ \\
& +\frac{\mathrm{G}}{H^{\mathrm{O}}(\Omega)} \iint_{\Omega^{\prime}}^{\mathrm{R}+H^{\mathrm{O}}\left(\Omega^{\prime}\right)} \delta \rho\left(r^{\prime}, \Omega^{\prime}\right)\left(\ell^{-1}\left(\mathrm{R}, \Omega ; r^{\prime}, \Omega^{\prime}\right)-\right. \\
& \left.-\ell^{-1}\left[r_{t}(\Omega) ; r^{\prime}, \Omega^{\prime}\right]\right) r^{\prime 2} \mathrm{~d} r^{\prime} \mathrm{d} \Omega^{\prime} .
\end{aligned}
$$

Substitution of Eqns. (19) and (20) back to Eq. (16) yields

$\bar{g}^{t}(\Omega)-g^{t}\left[r_{t}(\Omega)\right]+2 \pi \mathrm{G} \rho_{\mathrm{o}} H^{\mathrm{O}}(\Omega)=\bar{g}^{\mathrm{SBS}}(\Omega)-$ $-g^{\mathrm{SBS}}\left[r_{t}(\Omega)\right]+2 \pi \mathrm{G} \rho_{\mathrm{o}} H^{\mathrm{O}}(\Omega)+$ $+\bar{g} \operatorname{STC}(\Omega)-g \operatorname{STC}\left[r_{t}(\Omega)\right]+\bar{g} \delta \rho(\Omega)-g \delta \rho\left[r_{t}(\Omega)\right]$.

As follows from Eq. (22), the effects of anomalous density and terrain on the mean gravity absent in Poincaré-Prey's gravity gradient, i.e., $\partial g^{\text {STC }}(r, \Omega) / \partial H \approx 0$ and $\partial g^{\delta \rho}(r, \Omega) / \partial H \approx 0$. It yields the corrections to Helmert's orthometric height related to these two quantities.

The correction to Helmert's orthometric height due to the anomalous topographical density distribution $\varepsilon_{H}^{\delta \rho}(\Omega)$ reads

$\varepsilon_{H}^{\delta \rho}(\Omega)=-\frac{H^{\mathrm{O}}(\Omega)}{\bar{g}(\Omega)}\left(\bar{g}^{\delta \rho}(\Omega)-g^{\delta \rho}\left[r_{t}(\Omega)\right]\right)$.

Inserting expressions from Eqns. (18) and (21) into Eq. (23), this correction becomes

$\varepsilon_{H}^{\delta \rho}(\Omega) \cong-\frac{\mathrm{G}}{\gamma_{o}(\phi)} \iint_{\Omega^{\prime}} \int_{r^{\prime}=\mathrm{R}}^{\mathrm{R}+H^{\mathrm{O}}\left(\Omega^{\prime}\right)} \delta \rho\left(r^{\prime}, \Omega^{\prime}\right) \times$

$\times\left(\ell^{-1}\left(\mathrm{R}, \Omega ; r^{\prime}, \Omega^{\prime}\right)-\ell^{-1}\left[r_{t}(\Omega) ; r^{\prime}, \Omega^{\prime}\right]+\right.$

$\left.+\left.H^{\mathrm{O}}(\Omega) \frac{\partial \ell^{-1}\left(r, \Omega ; r^{\prime}, \Omega^{\prime}\right)}{\partial r}\right|_{r=r_{t}(\Omega)}\right) r^{\prime 2} \mathrm{~d} r^{\prime} \mathrm{d} \Omega^{\prime}$,

where the mean gravity $\bar{g}(\Omega)$ in the denominator of Eq. (24) is, in accordance with Eqn. (12), approximated by the normal gravity $\gamma_{o}(\phi)$.

The correction to Helmert's orthometric height due to the mean spherical terrain correction within the topography $\varepsilon_{H}^{\mathrm{STC}}(\Omega)$ is given by

$\varepsilon_{H}^{\mathrm{STC}}(\Omega)=-\frac{H^{\mathrm{O}}(\Omega)}{\bar{g}(\Omega)}\left(\bar{g}^{\mathrm{STC}}(\Omega)-g^{\mathrm{STC}}\left[r_{t}(\Omega)\right]\right)$.

By analogy with Eq. (24), Eq. (25) is rewritten as

$\varepsilon_{H}^{\mathrm{STC}}(\Omega) \cong$

$-\frac{\mathrm{G} \rho_{\mathrm{o}}}{\gamma_{o}(\phi)} \iint_{\Omega^{\prime}} \int_{r^{\prime}=\mathrm{R}+H^{\mathrm{O}}(\Omega)}^{\mathrm{R}+H^{\mathrm{O}}\left(\Omega^{\prime}\right)}\left(\ell^{-1}\left(\mathrm{R}, \Omega ; r^{\prime}, \Omega^{\prime}\right)-\ell^{-1}\left[r_{t}(\Omega) ; r^{\prime}, \Omega^{\prime}\right]+\right.$

$\left.+\left.H^{\mathrm{O}}(\Omega) \frac{\partial \ell^{-1}\left(r, \Omega ; r^{\prime}, \Omega^{\prime}\right)}{\partial r}\right|_{r=r_{t}(\Omega)}\right) r^{\prime 2} \mathrm{~d} r^{\prime} \mathrm{d} \Omega^{\prime}$.

In solving Newton's integral of Eqns. (24) and (26), the surface integration domain can be split into the near and far-zones. The numerical integration is then employed over the near-zone, while the far-zone contribution is negligible. Optimal size of the near-zone depends on the terrain and topographical density distribution around the computation point. The accuracy of numerical integration at the intermediate neighborhood of the computation point is unstable due to the weak singularity of Newton's kernel. Therefore, the topography can be discretized by the prism elements assuming the 
planar approximation of the geoid surface at the intermediate neighborhood of the computation point. The computation is then realized according to the formulae for the gravitational potential and attraction of prism summarized in [21].

The corrections to Helmert's orthometric height due to the anomalous topographical density and mean spherical terrain correction were computed for the same area as specified in the previous paragraph. At this territory, the orthometric heights range from 4 up to $2736 \mathrm{~m}$, and the anomalous laterally varying topographical density is between $-0,18$ and 0,31 g.cm ${ }^{-3}$. The correction to Helmert's orthometric height due to the anomalous topographical density was estimated to range between -6 and $3 \mathrm{~cm}$. Since data for radial density distribution were not available, the lateral density model was only used for the numerical integration of Eq. (24). This correction is often computed according to the simple formula $\varepsilon_{H}^{\delta_{\rho}}(\Omega) \approx 2 \pi \mathrm{G} \delta \rho(\Omega) \gamma_{o}^{-1}(\phi)\left[H^{\mathrm{o}}(\Omega)\right]^{2} \quad$ [4], Eqns. (4-34, 4-35). Comparing the results obtained from the above equation and Eq. (24), this approximation can be used everywhere except in the mountainous regions with the variable geological structure. In the mountains, the inaccuracy due to this approximation can even reach up to a few centimeters.

The correction to Helmert's orthometric height due to the mean spherical terrain correction is strongly correlated to the terrain. In the mountains, the correction is mostly negative and reaches up to $-17 \mathrm{~cm}$, while being positive in the valleys. The mean value of the spherical terrain correction in Eq. (25) was computed as the difference of the gravitational potentials of the terrain roughness. Niethammer and Mader's methods are compared to this method in the next paragraph.

From comparison of the mean gravitational attraction $\bar{g}^{\mathrm{SBS}}(\Omega)$ generated by the spherical Bouguer shell, which is given by the first term on the right-hand side of Eq. (21), and the corresponding expression $g^{\mathrm{SBS}}\left[r_{t}(\Omega)\right]-2 \pi \mathrm{G} \rho_{\mathrm{o}} H^{\mathrm{O}}(\Omega)$ of Poincaré-Prey's gravity reduction, the following second-order term is finally obtained

$$
\begin{aligned}
& -\frac{H^{\mathrm{O}}(\Omega)}{\bar{g}(\Omega)}\left(\bar{g}^{\mathrm{SBS}}(\Omega)-g^{\mathrm{SBS}}\left[r_{t}(\Omega)\right]+2 \pi \mathrm{G} \rho_{\mathrm{o}} H^{\mathrm{O}}(\Omega)\right) \cong \\
& \cong-\frac{8 \pi \mathrm{G} \rho_{\mathrm{o}}}{3 \gamma_{o}(\phi) \mathrm{R}}\left[H^{\mathrm{O}}(\Omega)\right]^{3} .
\end{aligned}
$$

Disregarding this term, the inaccuracy of orthometric height is less than $-1 \mathrm{~mm}$ for heights up to $3500 \mathrm{~m}$. For the height $8000 \mathrm{~m}$ this inaccuracy reaches $1,2 \mathrm{~cm} \mathrm{[9].}$

\section{Mader and Niethammer's mean terrain correction}

In order to improve the accuracy of Helmert's orthometric height, Niethammer [2] introduced the mean value of terrain correction along the plumbline $\bar{g}$ STC $(\Omega)$ to Poincaré-Prey's gravity reduction considering the constant topographical density distribution $\rho_{0}$. The approximate value $\tilde{g}^{\mathrm{N}}(\Omega)$ of mean gravity is then evaluated according to the following equation

$\tilde{g}^{\mathrm{N}}(\Omega) \approx \tilde{g}(\Omega)-g^{\mathrm{STC}}\left[r_{t}(\Omega)\right]+\bar{g}^{\mathrm{STC}}(\Omega)$.

As follows from Eq. (28), the point value ${ }_{g}$ STC $\left[r_{t}(\Omega)\right]$ of terrain correction computed for a point at the earth's surface is subtracted from Poincaré-Prey's gravity reduction and its mean value $\bar{g}^{\mathrm{STC}}(\Omega)$ is added instead.

The point values of the terrain correction $g^{\mathrm{STC}}(r, \Omega)$ are computed at the finite number $N$ of points along the plumbline within the topography. The mean value is then obtained by simple averaging

$$
\begin{gathered}
\bar{g}^{\mathrm{STC}}(\Omega) \approx \frac{1}{N} \sum_{n=1}^{N} g^{\mathrm{STC}}\left(r_{n}, \Omega\right), \quad n \in\langle 1,2, \ldots, N\rangle ; \\
r_{n} \equiv \mathrm{R}+n \Delta H ; \Delta H=H^{\mathrm{O}}(\Omega) / N,
\end{gathered}
$$

where $\Delta H$ is the step of the numerical integration with respect to height.

Under the assumption that the terrain correction varies linearly with depth, its mean value can approximately be estimated as the average of two values of the terrain correction $g$ STC $\left[r_{t}(\Omega)\right]$ and $g$ STC $\left[r_{g}(\Omega)\right]$ evaluated for a point at the earth's surface and geoid [3]. Thereby

$$
\tilde{g}^{\mathrm{M}}(\Omega) \approx \tilde{g}(\Omega)+\frac{g^{\mathrm{STC}}\left[r_{g}(\Omega)\right]-g^{\mathrm{STC}}\left[r_{t}(\Omega)\right]}{2} .
$$

In Niethammer and Mader's definitions, the planar form of the gravimetric terrain correction is usually considered, while its spherical form was used in this study. The reason follows from the fact that the interpretation of Poincaré-Prey's gravity reduction (Eq. 6) in the meaning of the infinite Bouguer plate is not rigorous.

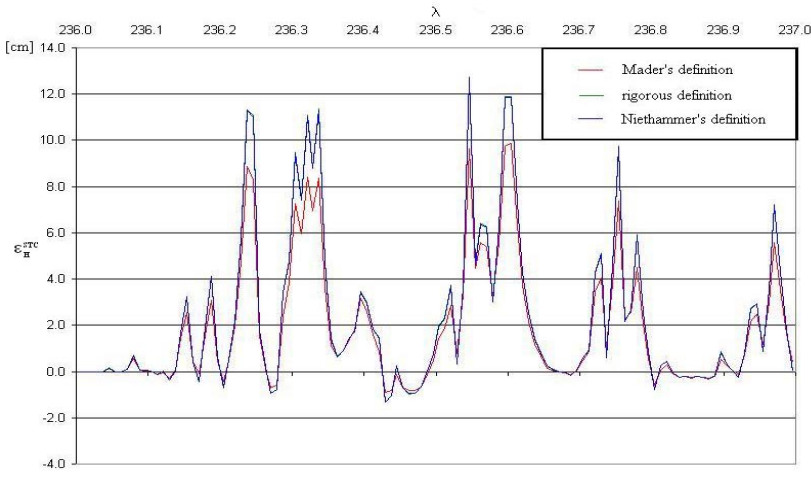

Comparison of different methods for evaluation of the mean terrain correction 
The numerical results of Niethammer and Mader's methods vere compared to the integral mean of the spherical terrain correction defined in terms of gravitational potential difference. As shown in Fig, Niethammer's method serves very similar results as the potential difference while the results of Mader's method are different. These differences caused by nonlinearity of the spherical terrain correction reach up to $2.5 \mathrm{~cm}$ in the mountains. On the other hand, the small differences between results of Niethammer's method and potential difference are caused by the discretization errors of Eq. (29). In this study, the $25 \mathrm{~m}$ step of the numerical integration was implemented with resulting subcentimetre relative accuracy.

\section{Summary and Conclusions}

Comparing the integral mean of gravity and Poincaré-Prey's gravity reduction, the corrections to Helmert's orthometric height are formulated. The main corrections are due to the mean geoid-generated gravity disturbance, mean spherical terrain correction and anomalous topographical density distribution. Regarding the correlation of these corrections with the topography, it follows that with the accuracy of a few centimeters, Helmert's orthometric heights are sufficiently accurate everywhere except in the mountains. Applying the corrections here, Helmert's orthometric heights can be improved in order of centimeters and decimeters. At the area of study, the complete contribution of the corrections to Helmert's orthometric height ranges between -22 and $4 \mathrm{~cm}$.

As the results indicate, Helmert's orthometric heights are usually smaller than the actual orthometric heights. The reason is that the dominant influence of the mean spherical terrain correction has a negative sign especially in the mountains. On the other hand, the correction to Helmert's orthometric height due to the mean geoid-generated gravity disturbance is mostly positive. It is worthwhile to mention that these conclusions are valid only for the computation area used in this study. Generally, the effect of the geoid-generated gravity disturbances can be significantly large. The correction due to the anomalous topographical density distribution can be negative as well as positive, depending on the geological structure of the region, i.e., the variation of the anomalous topographical density around the mean value $\rho_{0}$. The results provided in this paper are similar to those presented in [9], where the analytical downward continuation of the observed gravity was used to determine the corrections to Helmert's orthometric height. Considering the laterally varying topographical density, the approach used in this study is equivalent to the analytical downward continuation used in [9]. Therefore, the small differences of these results up to a few centimeters are caused by the different numerical accuracy of methods applied for the computation.

The correction to Helmert's orthometric height caused by the second-order term of the mean gravitational attraction of spherical Bouguer shell (given by Eq. 27) has a similar magnitude to and the opposite sign to the correction due to the variation of the normal gravity gradient (Eq. 11). Thereby, their total effect on the orthometric heights is less than $1 \mathrm{~mm}$ for heights up to $8000 \mathrm{~m}$.

As discussed in paragraph 7, Niethammer's method serves numerically similar result as the integral mean of the spherical terrain correction defined in terms of the gravitational potential difference. On the other hand, the result of Mader's method differs from the previous two methods. The inaccuracy of Mader's method reaches up to $2,5 \mathrm{~cm}$ in the mountains, where the mean terrain correction is systematically smaller. This is not in accordance with conclusions presented by Mader [3] and Ledersteger [22]. They obtained very similar results to Niethammer's approach on a sub-centimetre level, which confirms the assumption of linear change of the terrain correction with depth. On the other hand, results in paragraph 7 shows that the assumption of linearity cannot be taken into account. This disagreement is caused by using spherical instead of planar form of the terrain correction.

Concluding from the above results, the orthometric heights can be determined with a reasonable accuracy to provide the meaningful information about the physical shape of the Earth.

Acknowledgement: The detailed digital terrain model used in this research was kindly provided by the National Resources Canada. The numerical comparison of different methods for the evaluation of the mean terrain correction along the plumbline prepared by Robert Kingdom from the University of New Brunswick is also gratefully acknowledged.

\section{References}

1. Helmert, F. R. Die Schwerkraft im Hochgebirge, insbesondere in den Tyroler Alpen. Veröff. Königl. Preuss. Geod. Inst., No 1, 1890.

2. Niethammer, T. Nivellement und Schwere als Mittel zur Berechnung wahrer Meereshöhen. Schweizerische Geodätische Kommission, 1932.

3. Mader, K. Die orthometrische Schwerekorrektion des Präzisions-Nivellements in den Hohen Tauern. Österreichische Zeitschrift für Vermessungswesen, Sonderheft 15, 1954.

4. Heiskanen, W. H.; Moritz, H. Physical geodesy. WH Freeman and Co., San Francisco, 1967.

5. Vaníček, P.; Kleusberg, A.; Martinec, Z.; Sun, W.; Ong, P.; Najafi, M.; Vajda, P.; Harrie, L.; Tomášek, P.; Horst, B. Compilation of a precise regional geoid. Final report on research done for the Geodetic Survey Division, Fredericton, 1995.

6. Allister, N. A.; Featherstone, W. E. Estimation of Helmert orthometric heights using digital barcode leveling, observed gravity and topographic mass-density data over part of Darling Scarp, western Australia. Geomatics Research Australasia, Vol. 75, 2001, p. 25-52.

7. Tenzer, R.; Vaníček, P. Correction to Helmert's orthometric height due to actual lateral variation of topographical density. Brazilian Journal of Cartography 
(Revista Brasileira de Cartografia), 55(02), 2004, p. 4447.

8. Hwang, C.; Hsiao, Y. S. Orthometric height corrections from leveling, gravity, density and elevation data: a case study in Taiwan. J Geod, 77 (5-6), 2003, p. 292-302.

9. Tenzer, R.; Vaníček, P.; Santos, M. Corrections to Helmert's Orthometric Heights. Proceedings of IUGG symposia, Sapporo, Japan, July 9-10, (in print) 2005.

10. Tenzer, R.; Vaníček, P.; Santos, M.; Featherstone, W. E.; Kuhn, M. The rigorous determination of orthometric heights. J Geod (accepted) 2005.

11. Pizzetti, P. Sopra il calcolo teorico delle deviazioni del geoide dall ellissoide. Atti R. Accad. Sci. Torino, V. 46, 1911.

12. Somigliana, C. Teoria Generale del Campo Gravitazionale dell'Ellisoide di Rotazione. Memoire della Societa Astronomica Italiana, IV, Milano, 1929.

13. Bruns, H. Die Figur der Erde. Publ. Preuss. Geod. Inst., Berlin, 1878.

14. Vaníček, P.; Tenzer, R.; Sjöberg, L. E.; Martinec, Z.; Featherstone, W. E.; New views of the spherical Bouguer gravity anomaly. Geoph J Int 159(2), 2004, p. 460-472.

15. Bomford, G. Geodesy, $3^{\text {rd }}$ edition. Clarendon Press, Oxford, 1971

16. Molodensky, M. S. Fundamental Problems of Geodetic Gravimetry. Trudy NIIGAIK, Vol. 42, Geodezizdat, Moscow, 1945 (in Russian).

17. Martinec, Z. Boundary value problems for gravimetric determination of a precise geoid. Lecture notes in earth sciences, Vol. 73, Springer 1998.

18. Wichiencharoen, $\mathrm{C}$. The indirect effects on the computation of geoid undulations. Dept of Geod Sci Report, No 336, Ohio State University, 1982.

19. Martinec, Z.; Vaníček, P. Direct topographical effect of Helmert's condensation for a spherical approximation of the geoid. Manusc Geod, 19, 1994, p. 257-268.

20. Huang, J.; Vaníček, P.; Pagiatakis, S. D.; Brink, W. Effect of topographical density on the geoid in the Rocky Mountains. J Geod, 74, 2001, p. 805-815.

21. Nagy, D.; Papp, G.; Benedek, J. The gravitational potential and its derivatives for the prism. Journal of Geodesy, 74 (7/8), 2000, p. 552-560.

22. Ledersteger, K. Der Schwereverlauf in den Lotlinien und die Berechnung der wahren Geoidschwere. Publication dedicated to W.A. Heiskanen, Publ. Finn. Geod. Inst., No 46, 1955, p. 109-124.
Robert TENZER. Ing, PhD, Research Associate.

School of Civil Engineering and Geomatics, University of Newcastle upon Tyne, Cassie Building, Newcastle upon Tyne, NE1 7RU, United Kingdom (Tel: +44 (0)191 222 8202; Fax: +44 (0)191 222 6399); e-mail: robert.tenzer@ncl.ac.uk.

A graduate of the Slovak Technical University, Bratislava (engineer of geodesy, 1995) and herein obtained $\mathrm{PhD}$ degree in 2000. Currently is studding at the Czech Technical University in Prague. Teaching experience: Departments of Informatics and Geography at the Matej Bel University (1997-1999) and Department of Geodesy at the University of Zilina (19992001). Research experience: Topographical Institute of Slovak Republic (1995-1996), Department of Geomatics at the University of New Brunswick (2001-2004), and School of Civil Engineering and Geomatics at the University of Newcastle upon Tyne (from 2005). Author or co-author of more than 60 scientific papers.

Research topics: satellite geodesy, physical geodesy. 